\title{
C/EBPZ modulates the differentiation and proliferation of preadipocytes
}

\author{
Yuechan Chen ${ }^{1,2,4}$, Lingyu Gao ${ }^{1,2,4}$, Tao Lin ${ }^{1,2}$, Xuelian Pei ${ }^{1,2,3}$, Qin Gao ${ }^{1,2}$, Jingjing Chen ${ }^{1,2}$, Yi Zhang ${ }^{1,2}$, Xiangqi Wu ${ }^{1,2}, Z^{1}$ equan Li ${ }^{1,2}$ and \\ Zhiwei Zhang $\mathbb{D}^{1,2,3}$ 凶
}

(C) The Author(s), under exclusive licence to Springer Nature Limited 2021

BACKGROUND/OBJECTIVES: This study investigated the functions of CCAAT/enhancer-binding protein zeta (C/EBPZ; Gene ID: 10153) in adipose tissue.

SUBJECTS/METHODS: Bioinformatics analysis were used to study the expression pattern of C/EBPZ in human adipose tissue. The expression and function of $C / E B P Z$ in adipose tissue were further studied using chicken as animal model in vivo and in vitro. RESULTS: The human $C / E B P Z$ transcripts were greater and more stable in subcutaneous adipose tissue than in visceral adipose tissue $(P<$ $0.01)$, and they were increased with age in adipose tissue $(P<0.05)$. In addition, the chicken $C / E B P Z$ transcripts $(C / E B P Z / A C T B)$ of visceral (abdominal) adipose tissue were significantly different between fat and lean broilers and decreased with age during development $(P<$ 0.01). RNA-seq analysis showed that the C/EBPZ overexpression associated with adipose tissue development and DNA replication in chicken preadipocytes $(P<0.05)$. Additionally, overexpression of chicken $\mathrm{C} / \mathrm{EBPZ}$ inhibited preadipocytes differentiation and promoted preadipoytes proliferation in vitro $(P<0.05)$. In addition, $C / E B P Z$ overexpression suppressed the promoter activities of $P P A R \gamma, C / E B P a, F A S N$ and $L P L$, and promoted the promoter activities of GATA2 and FABP4 in chicken preadipocytes $(P<0.05)$.

CONCLUSIONS: C/EBPZ modulated the differentiation and proliferation of preadipocytes, and it might be a new negative regulator of adipogenesis.

International Journal of Obesity (2022) 46:523-534; https://doi.org/10.1038/s41366-021-01020-z

\section{INTRODUCTION}

Adipose tissue, a loose connective tissue composed mostly of adipocytes, is an important organ for lipid storage and endocrine in human being and animals [1]. Abnormality in formation of adipose tissue results in obesity, metabolic syndrome and lipodystrophy, and it is associated with type 2 diabetes mellitus (T2DM), cardiovascular disease, nonalcoholic fatty liver disease, cancers, and other several diseases [2].

Adipogenesis is mediated by a series of complex processes, including commitment of mesenchymal stem cells into preadipocytes and differentiation of preadipocytes to mature adipocytes [3]. Transcriptional control plays an important role in adipogenesis [3]. Of transcription factors (TFs), peroxisome proliferator-activated receptor gamma (PPARY) is undoubtedly the most important transcriptional modulator of adipocyte development in all types of adipose tissue [4]. In addition, CCAAT/enhancer-binding proteins (C/EBPs), Kruppel-like factors (KLFs) and some other TFs play important roles in adipogenesis [4].

CCAAT/enhancer-binding protein zeta (C/EBPZ, Gene ID: 10153); alternative names CBF, CBF-2, NOC1 and HSP-CBF, is a ubiquitous and highly conserved protein in animals. Despite its name it should not be considered as a C/EBP family member because it lacks significant homology with other C/EBP members, and in fact it is not a basic leucine zipper (bZIP) protein [5]. To complicate things even more, DNA damage inducible transcript 3 (DDIT3, Gene ID: 1649) was also named C/EBPZ in several publications [6].

The real C/EBPZ (Gene ID: 10153) was first reported as a CCAATbox binding transcription factor of human heat-shock protein 70 (HSP70) promoter, and it facilitated HSP70 promoter activity in a CCAAT-dependent manner [7]. Further study showed that C/EBPZ might not directly DNA sequence-specific bind to CCAAT-box but associated with a CCAAT-bound NF-Y in HSP activation, and it could be a protein scaffold that integrates interactions with different transcription factors [8].

C/EBPZ was reported to be a mRNA binding protein $[9,10]$ and nucleolar protein [11] in human cells. Additionally, C/EBPZ was involved in m6A methylation of RNA by recruiting methyltransferase 3, N6-adenosine-methyltransferase complex catalytic subunit (METTL3) to transcription start sites (TSSs) of target genes in acute myeloid leukemia (AML) [12].

Genetic study showed that $C / E B P Z$ was a recurrently mutated gene in AML [13] and associated with schizophrenia in human [14]. Additionally, comparative host-coronavirus protein interaction networks revealed that C/EBPZ might take a part in the

\footnotetext{
${ }^{1} \mathrm{NHC}$ Key Laboratory of Prevention and Treatment of Central Asia High Incidence Diseases of First Affiliated Hospital, School of Medicine, Shihezi University, Shihezi, China. ${ }^{2}$ Key Laboratory of Xinjiang Endemic and Ethnic Diseases of Ministry of Education, School of Medicine, Shihezi University, Shihezi, China. ${ }^{3}$ Department of Histology and Embryology,
} School of Medicine, Shihezi University, Shihezi, China. ${ }^{4}$ These authors contributed equally: Yuechan Chen, Lingyu Gao. ${ }^{\circledR e m a i l: ~ z z w n e a u @ 163 . c o m ~}$ 
524

viral-human protein-protein interaction for SARS-CoV-1, MERSCoV, and SARS-CoV-2 [15].

DDIT3 (Gene ID: 1649), once named C/EBPZ in several publications, was reported as an important negative regulator in adipogenesis [16], however, there was no report of real C/EBPZ (Gene ID: 10153) on adipogenesis to date. In the current study, the role of C/EBPZ (Gene ID: 10153) in the regulation of adipogenesis was studied, and the results provided an evidence that C/EBPZ was a new negative regulator of adipogenesis.

\section{MATERIALS AND METHODS Experimental birds and management}

All animal experiments were conducted according to the guidelines for care and use of experimental animals established by the Ministry of Science and Technology of the People's Republic of China (Approval number: 2006-398), and they were approved by the Animal Experimental Ethical Committee of First Affiliated Hospital, Shihezi University School of Medicine (Approval number: A2019-009-01). In brief, several 12-day-old and six 7-week-old male Arbor Acres (AA) broilers were sacrificed under ether anaesthesia for stromal-vascular cells isolation and tissue expression analysis, respectively, and before sacrificed, they all had free access to feed and water.

\section{Tissues}

Six male AA birds were slaughtered at 7 weeks of age, 15 tissue samples were collected: including, visceral (abdominal) fat tissue, liver, duodenum, jejunum, ileum, pectoralis, leg muscle, gizzard, heart, spleen, kidney, pancreas, proventriculus, brain, and testis. All the tissues collected were snap-frozen and stored in liquid nitrogen until RNA extraction.

Information regarding the abdominal adipose tissues of the 14th generation population of Northeast Agricultural University broiler lines divergently selected for abdominal fat content (NEAUHLF; fat and lean line, each line 53 male broilers) had been published previously $[17,18]$, and they were kindly gift from professor Hui Li of Northeast Agricultural University. Briefly, after 14 generations of divergent selection for abdominal fatness, the abdominal fat percentage at 7 weeks of age in the fat broiler line was 4.45 times that of the lean line. The experimental groups were not randomized, but were divided into fat and lean groups based on the 14 generations of divergent selection for abdominal fat content. In addition, a sample size of 53 in each group achieved a confidence level of $91 \%$ to detect the difference in C/EBPZ expression between fat and lean birds. The experimenters were not blinded to groups on the test days, however, most of the subsequent analyses were performed in blinded conditions.

\section{Vector construction}

To clone the coding sequence (CDS) of chicken $C / E B P Z$, total RNA from abdominal fat tissue of 7-week-old AA broilers was reverse-transcribed using oligo(dT)-anchor primer and ImProm-II reverse transcriptase (Promega, Madison, USA). A set of primers, C/EBPZ -F1 (5'- GCGTCGACC ATGGCGGCGCTCGGGGAGT-3') and C/EBPZ -R1 (5'- GAAGATCTTCATCTITT TGATTTCTTGCCTC-( ${ }^{\prime}$ ), was designed according to the chicken C/EBPZ sequences (GenBank accession NM_001031060.1). Additionally, LA Taq (TaKaRa, Dalian, China) was used to perform PCR reaction. After gel extraction, the PCR product of C/EBPZ was cloned into the pMD-18T vector (TaKaRa) for sequencing (BGl, Beijing, China). To generate the $C / E B P Z$ overexpression vector (pCMV-HA-C/EBPZ), the C/EBPZ CDNA fragment, produced by digestion of pMD-18T-C/EBPZ using Sall and Bglll (TaKaRa), was subcloned into the pCMV-HA vector (Clontech, Mountain View, USA).

The promoter-reporter plasmids of chicken PPARY, C/EBPa, fatty acid synthase (FASN), adipocyte fatty acid-binding protein (FABP4) and Lipoprotein lipase (LPL) were described in literatures [19]. The promoterreporter plasmid of chicken GATA binding protein 2 (GATA2), pGL4.10GATA2 (-2 001/-1), is a luciferase reporter plasmid of pGL4.10 (Promega) containing the chicken GATA2 promoter (nucleotides $-2001 \mathrm{bp}$ to $-1 \mathrm{bp}$, relative to the start site of NM_001003797.1), and the promoter-reporter plasmid of chicken GATA binding protein 3 (GATA3), pGL4.10-GATA3 (-296/ +186 ), is the luciferase reporter plasmid of pGL4.10 (Promega) containing the chicken GATA3 promoter (nucleotides $-296 \mathrm{bp}$ to $+186 \mathrm{bp}$, relative to the start site of X66844.1). Both of them were constructed by full-length sequence synthesis of BGI company.

\section{Cell culture}

Chicken stromal-vascular cells were isolated from 12-day-old chickens according to the procedure previously described [19], and the separated chicken stromal-vascular cells were considered as chicken preadipocytes and grown in DMEM/F12 medium supplemented with $10 \%$ fetal bovine serum and maintained at $37{ }^{\circ} \mathrm{C}$ in a humidified, $5 \% \mathrm{CO}_{2}$ atmosphere. Before function study, all the cells had been treated with TransSafe ${ }^{\mathrm{TM}}$ Mycoplasma Elimination Reagent (TransMyco Plus) Kit (TransGen, Beijing, China) to remove mycoplasma contamination. The experimental groups of cells were randomized before transfection or induction by oleate, and the experimenters were blinded to groups on the test days.

\section{RNA isolation and real-time reverse transcription-PCR}

Total RNA was extracted from stored tissues $(100 \mathrm{mg})$ or chicken preadipocytes using Trizol (Invitrogen, Carlsbad, USA) following the manufacturer's protocol. RNA quality was assessed by denaturing formaldehyde agarose gel electrophoresis. Reverse transcription (RT) was performed using $1 \mu \mathrm{g}$ of total RNA, an oligo(dT) anchor primer (Promega), and ImProm-II reverse transcriptase (Promega), and reverse transcription conditions were $25^{\circ} \mathrm{C}$ for $5 \mathrm{~min}, 42^{\circ} \mathrm{C}$ for $60 \mathrm{~min}$, and $70^{\circ} \mathrm{C}$ for $15 \mathrm{~min}$.

Real-time PCR was used to detect gene expression using the SYBR Premix Ex Taq (Takara) on a 7500 Real-Time PCR System (Applied Biosystems, Foster City, USA) with the primers shown in Table 1. Chicken glyceraldehyde 3-phosphate dehydrogenase (GAPDH) or beta-actin (ACTB) were used as internal references. Part $(1 \mu \mathrm{L})$ of each RT reaction product was amplified in a $20-\mu \mathrm{L} P C R$ reaction system. Reaction mixtures were incubated in an $\mathrm{ABI}$ Prism 7500 sequence detection system (Applied Biosystems), programmed to conduct 1 cycle at $95^{\circ} \mathrm{C}$ for $30 \mathrm{~s}$ and 40 cycles at $95^{\circ} \mathrm{C}$ for $5 \mathrm{~s}$ and $60^{\circ} \mathrm{C}$ for $34 \mathrm{~s}$. Moreover, dissociation curves were analyzed using Dissociation Curve 1.0 software (Applied Biosystems) for each $\mathrm{PCR}$ reaction to detect and eliminate possible primer-dimer artifacts. All reactions were performed in triplicate. The relative amounts of C/EBPZ transcripts were calculated using the comparative cycle-time method.

\section{Western blot assays}

Chicken preadipocytes transfected with pCMV-HA-C/EBPZ or pCMV-HA for 2 days were lysed in RIPA buffer (PBS, pH 7.4, containing 1\% NP-40, 0.5\% sodium deoxycholate, $0.1 \%$ SDS, and a protease inhibitor cocktail). Then, the cell lysates were added into $5 \times$ denaturing loading buffer and boiled in water for $5 \mathrm{~min}$. Cell lysates were separated on 5-12\% SDS-polyacrylamide gels and transferred to polyvinylidenedifluoride membranes. After incubation with the primary antibody for HA-tag [HA-tag (C29F4) rabbit mAB \#3742, CST, Danvers, USA; 1:1 000], chicken ACTB (anti- $\beta$ actin mAB \#TA-09, ZSGB-BIO, Beijing, China;1:1 000) or chicken PPARy [PPARy (81B8) Rabbit mAb \#2443, CST;1:1 000], a secondary horseradish peroxide-conjugated antibody (1:5 000; Beyotime, Beijing, China) was added, and a BeyoECL Plus kit (Beyotime) was used for detection.

Table 1. Primers used for real-time PCR.

\begin{tabular}{|c|c|c|}
\hline Genes Name & Sequence ID & Primers $\left(5^{\prime}-3^{\prime}\right)$ \\
\hline \multirow[t]{2}{*}{ HSPB8 } & XM_004934409 & GAGGAGCTGACGGTCAAAAC \\
\hline & & AAGACAGTGATGGGGTCCAC \\
\hline \multirow[t]{2}{*}{ GIT1 } & NM_204296 & CTCAGGCCAACTTCTTCCAC \\
\hline & & CAGCTCATACTGGCACTCCA \\
\hline \multirow[t]{2}{*}{ KLF2 } & JQ687128 & ATACCATCCTGCCCTCCTTC \\
\hline & & CTGCCCATGGAAAGGATAAA \\
\hline \multirow[t]{2}{*}{ ACTB } & NM_205518 & TCTTGGGTATGGAGTCCTG \\
\hline & & TAGAAGCATTTGCGGTGG \\
\hline \multirow[t]{2}{*}{$C / E B P Z$} & NM_001031060 & GGCCCAGACCTTAACAATGA \\
\hline & & GTCAAACTTGGACCCAGCAT \\
\hline \multirow[t]{2}{*}{ PPARY } & NM_001001460 & CAACTCACTTATGGCTA \\
\hline & & СTTATTTCTGCTTTTCT \\
\hline \multirow[t]{2}{*}{ GAPDH } & NM_204305 & CTGTCAAGGCTGAGAACC \\
\hline & & GATAACACGCTTAGCACCA \\
\hline
\end{tabular}


Table 2. The plasmid system transfected in one well of 12-well dishes.

\begin{tabular}{lllll} 
Group & Reporter plasmid & & pRL-TK & C/EBPZ-Overexpression plasmid $^{\mathbf{a}}$ \\
\hline PPARY & pGL3-basic-PPARY $(-1978 /-82)$ & $400 \mathrm{ng}$ & $8 \mathrm{ng}$ & $600 \mathrm{ng}$ \\
\hline C/EBPa & pGL3-basic-C/EBPa $(-1863 /+332)$ & $200 \mathrm{ng}$ & $10 \mathrm{ng}$ & $800 \mathrm{ng}$ \\
\hline FASN & pGL3-basic-FASN $(-1096 /+160)$ & $400 \mathrm{ng}$ & $8 \mathrm{ng}$ & $600 \mathrm{ng}$ \\
\hline LPL & pGL3-basic-LPL $(-1914 /+66)$ & $400 \mathrm{ng}$ & $20 \mathrm{ng}$ & $600 \mathrm{ng}$ \\
\hline FABP4 & pGL3-basic-FABP4 $(-1996 /+22)$ & $400 \mathrm{ng}$ & $20 \mathrm{ng}$ & $600 \mathrm{ng}$ \\
GATA2 & pGL4.10-GATA2 $(-2001 /-1)$ & $400 \mathrm{ng}$ & $8 \mathrm{ng}$ & $600 \mathrm{ng}$ \\
GATA3 & pGL4.10-GATA3 $(-296 /+186)$ & $400 \mathrm{ng}$ & $8 \mathrm{ng}$ & $600 \mathrm{ng}$
\end{tabular}

${ }^{\mathrm{a}}$ The plasmids used in the groups of C/EBPZ and EV were $\mathrm{pCMV}-\mathrm{HA}-\mathrm{C} / E B P Z$ and $\mathrm{pCMV}-\mathrm{HA}$ (empty vector, EV), respectively.

Table 3. Information of C/EBPZ genes used for sequence analysis.

\begin{tabular}{|c|c|c|c|c|}
\hline Species & Nucleotides & Location & Protein & Size of protein \\
\hline Human (Homo sapiens) & NM_005760 & chromosome $=$ "2" & NP_005751 & 1054 aа \\
\hline Chicken (Gallus gallus) & NM_001031060 & chromosome $=" 3 "$ & NP_001026231 & 1076 aа \\
\hline Mouse (Mus musculus) & NM_033563 & chromosome $=" 1 "$ & NP_001019977 & 1052 aа \\
\hline Rat (Rattus norvegicus) & NM_001108701 & chromosome $=" 6 "$ & NP_001102171 & 1045 aа \\
\hline Tropical clawed frog (Xenopus tropicalis) & NM_001127414 & chromosome $=" 5 "$ & NP_001120886 & 1003 aа \\
\hline Zebrafish (Danio rerio) & NM_001017745 & chromosome $=" 20 "$ & NP_001017745 & 1029 aa \\
\hline
\end{tabular}

\section{RNA-seq analysis}

The chicken preadipocytes transfected with $\mathrm{PCMV}-\mathrm{HA}-\mathrm{C} / E B P Z$ and empty vector ( $\mathrm{pCMV}-\mathrm{HA})$ for $48 \mathrm{~h}$, respectively, were collected using Trizol (Invitrogen) for RNA-seq analysis. The RNA-seq analysis and following data analysis were performed by Novogene company (Beijing, China).

\section{Induction of chicken preadipocyte differentiation}

Chicken preadipocytes were passaged and seeded into 6-well plates at a density of $1 \times 10^{5}$ cells $/ \mathrm{cm}^{2}$. After $12 \mathrm{~h}$, when the cells were $60-80 \%$ confluent, they were transfected with $\mathrm{pCMV}-\mathrm{HA}-\mathrm{C} / E B P Z$ or $\mathrm{pCMV}-\mathrm{HA}$ using the FUGENE HD transfection reagent (Promega) according to the manufacturer's recommendations. At $24 \mathrm{~h}$ after transfection, $160 \mu \mathrm{M}$ oleate (Sigma) was added into the medium to induce preadipocyte differentiation for $48 \mathrm{~h}$.

\section{Oil red $\mathrm{O}$ staining}

We followed the methods of Li et al. in 2014 [18]. Oil red O staining of intracellular lipid droplets was performed in 6-well plates. Chicken preadipocytes induced to differentiate for $48 \mathrm{~h}$ were washed with PBS and fixed in $10 \%$ formaldehyde for $10 \mathrm{~min}$. After rinsing with distilled water, they were stained with $0.5 \%$ oil red $\mathrm{O}$ working solution, prepared by vigorously mixing three parts of a stock solution $(0.5 \%$ oil red $\mathrm{O}$ in isopropanol; Sigma) with two parts of water for $5 \mathrm{~min}$ and filtering through a $0.4-\mu \mathrm{m}$ filter. Excess staining was removed by rinsing twice with PBS. The dye was extracted by $1 \mathrm{~mL}$ isopropanol incubation, and quantitative assessment was obtained by spectrophotometric analysis of absorbance of the extracted dye at $500 \mathrm{~nm}$.

\section{Cell proliferation assay}

After the chicken preadipocytes were transfected with $\mathrm{pCMV}-\mathrm{HA}-\mathrm{C} / \mathrm{EBPZ}$ and PCMV-HA, respectively, and incubation for $24 \mathrm{~h}$, these cells were passaged and seeded into 96 -well plates at a concentration of 5000 cells per well, respectively. At the designated time points after passage, including $24 \mathrm{~h}, 48 \mathrm{~h}, 72 \mathrm{~h}, 96 \mathrm{~h}$ and $120 \mathrm{~h}$, the cell viability was detected using CellTiter-Glo ${ }^{\circledR}$ Luminescent Cell Viability Assay (Promega) following the manufacturer's protocol.

\section{Flow cytometric analysis}

Chicken preadipocytes were seeded in 6-well plates. Transfection was performed when the cells were about $60 \%$ confluent. After transfection for $48 \mathrm{~h}$, cells were digested by trypsin, and then ceased the digestion using DMEM/F12 with $10 \%$ FBS. The cells were transferred to $15 \mathrm{~mL}$ microcentrifuge tubes and centrifuged under the condition of $1000 \mathrm{rpm}$ for
$5 \mathrm{~min}$ at $4^{\circ} \mathrm{C}$ to discard the supernatant. The remained cells were resuspended and washed by $1 \mathrm{~mL}$ cold PBS. Then PBS was discard after centrifugation of $1000 \mathrm{rpm}$ for $5 \mathrm{~min}$ at $4{ }^{\circ} \mathrm{C}$, and the cells were fixed in $100 \%$ ethanol overnight at $-20^{\circ} \mathrm{C}$. After that, the fixed cells were stained with Cell cycle staining Kit (MultiSciences, Hangzhou, China), and the flow cytometric analysis was performed using BD FACSAria ${ }^{\mathrm{TM}}$ III flow cytometer (BD Biosciences, San Jose, USA), and the data were analyzed using FlowJo software.

\section{Luciferase reporter assays}

The luciferase reporter assays were performed in chicken preadipocytes in 12-well dishes. In transfection assays, a fixed amount of total DNA $(1 \mu \mathrm{g})$ was transfected in each well using FuGENE HD transfection reagent (Promega). The transfection system for plasmids was shown in Table 2. After transfection and incubation for $48 \mathrm{~h}$, the cells were lysed in $250 \mu \mathrm{L}$ of 1-time passive lysis buffer (Promega), and portions of the lysate were subjected to assays for firefly and renilla luciferase using the DualLuciferase Reporter Assay System (Promega). Promoter activity of each construct was expressed as the ratio of Firefly / Renilla luciferase activity.

\section{Sequence analysis}

The sequences used were all from NCBI protein database. Because the predicted sequences might be updated frequently, only the sequences of C/EBPZ officially certified by NCBI and titled by 'NP_' were used for sequence analysis. The sequences used included six species, including human, mouse, rat, chicken, frog and zebrafish (Table 3). DNAMAN was employed to get the overall identity of the multiple sequence alignment, and Clustal Omega was used to get the percent identity matrix. The Genomicus software program (http://www.dyogen.ens.fr/genomicus62.02/cgi-bin/search.pl) was used to identify syntenic genes near C/EBPZ in the genomes of animals.

\section{Statistical analysis}

All statistical analyses were performed using the $\mathrm{R}$ software system (version R 3.5.1; https://cran.rproject.org/bin/windows/base/old/3.5.1/). Sample size estimation was performed using the $\mathrm{R}$ package 'pwr'. The Shapiro-Wilk test was used to test the normality of data. The difference analysis between two groups was performed by two tailed Student's $t$ test or Wilcoxon rank sum test. The difference analysis in more than two groups was analyzed by ANOVA or Kruskal-Wallis tests, and comparisons among more than two groups were performed by Duncan's multiple tests. The data that conformed to the normal distribution were directly applied to Duncan's multiple tests, and the data that did not conform to the normal 
distribution were sorted rank firstly, and the ranks were then applied to Duncan's multiple tests.

The chicken C/EBPZ transcripts of abdominal fat tissues from NEAUHLF were analyzed by Scherier-Ray-Hare tests with the following model:

$\mathrm{Y}=\mu+\mathrm{A}+\mathrm{L}+\mathrm{A}^{*} \mathrm{~L}+\mathrm{e}$

Where $Y$ is the dependent variable (the relative C/EBPZ expression), $\mu$ is the population mean, $A$ is the fixed effect of the age, $L$ is the line (broiler line selected by high and low abdominal fat content) as fixed effect, $A^{*} L$ is the interaction of age and line, and $\mathrm{e}$ is the random error.

The expression data of C/EBPZ of human adipose tissues (Supplementary Table 1) were achieved from the human protein atlas (https://www. proteinatlas.org/) [20] and analyzed by Cochran-Mantel-Haenszel test. The relationship between the PTMP of C/EBPZ and the percentage of adipocytes in adipose tissue was analyzed by Kendall rank test. The relationships between the pTMPs of C/EBPZ and other genes in adipose tissue were analyzed by Spearman tests using GEPIA online serves.

The data were presented as mean \pm standard deviations (SD) unless otherwise indicated, and differences were considered significant at $P<0.05$ unless otherwise indicated.

\section{RESULTS \\ Bioinformatics analysis of $C / E B P Z$ expression in human adipose tissue}

The data from human protein atlas showed that there was a low expression level of C/EBPZ in adipocytes (Fig. 1A), and the pTPM of $C / E B P Z$ in adipose tissue was negative related to the percentage of adipocytes ( $\mathrm{tb}=-1, P=0.0415$; Fig. $1 \mathrm{~B}$ ).

The pTPM data of C/EBPZ did not conform to a normal distribution (Shapiro-Wilk test; $W=0.99531, P=0.0156$ ). Cochran-Mantel-Haenszel test showed that C/EBPZ transcripts of human adipose tissue were significantly associated with the location $(H=6.4901, P=0.0108)$ and age $(H=18.2767, P=$ $0.0026)$, and there was no significant association of them with $\operatorname{sex}(H=0.4001, P=0.5270)$.

Wilcoxon rank sum test showed that the C/EBPZ transcripts of subcutaneous adipose tissue were significantly greater than those of visceral adipose tissue $(W=87646, P=0.004438$; Fig. 1D). Duncan's multiple tests showed that the C/EBPZ expression increased with age $(P<0.05$; Fig. $1 \mathrm{E})$. In addition, the $C / E B P Z$ transcripts of subcutaneous adipose tissue were significantly greater than those of visceral adipose tissue at the ages of 20-29 years and 30-39 years $(P<0.05$; Fig. 1F).

Additionally, Spearman correlation tests showed that the $C /$ $E B P Z$ transcripts of adipose tissue were negatively associated with those of PPAR $, C / E B P a, F A S N, L P L$, Perilipin 1 (PLIN1) and Sterol regulatory element binding transcription factor 1 (SREBP1) $(r<0$, $P<0.05)$, and they were positively associated with the transcripts of KLF7 and DDIT3 ( $r>0, P<0.05$, Supplementary Fig. 1).

Sequence and tissue expression pattern of chicken C/EBPZ Chicken $C / E B P Z$ was first reported in a study to sequence and characterize a large number of full-length cDNAs from bursal lymphocytes [21]. However, to date, there was no further report on chicken $C / E B P Z$. In this study, sequence analysis showed that there were 1076 amino acids in predicted chicken C/EBPZ, and it could express a protein about $123 \mathrm{kD}$. Chicken C/EBPZ has a high similarity in protein sequence to its human and murine orthologs ( $>60 \%$, amino acids identity, Table 4 ). Especially, the conserved domains related to the interaction of NF-Y trimer [8] and function of proribosome [22] also could be found in chicken C/EBPZ (Supplementary Fig. 2). In addition, C/EBPZ was in a conserved chromosome region during the evolution of animals, and the linkage group of CDC42EP3-QPCT-PRKD3-C20RF56-C/EBPZ could be detected in both human and chicken chromosomes (Supplementary Table 2).

Real-time RT-PCR analysis showed that $C / E B P Z$ was expressed ubiquitously in various chicken tissues at the age of 7 weeks, including duodenum, abdominal fat, brain, pectoralis, proventriculus, heart, ileum, kidney, jejunum, liver, leg muscle, gizzard, pancreas, spleen, and testis (Supplementary Fig. 3). The data of C/ EBPZ transcripts did not conform to a normal distribution (Shapiro-Wilk test; $W=0.647974, \quad P<0.0001)$. Kruskal-Wallis analysis showed that the $C / E B P Z$ transcripts were significantly associated with the category of tissues $(H=70.1, P<0.001)$. Duncan's multiple tests showed that among of them, pancreas, jejunum, testis and abdominal fat had high expression levels of $C$ / $E B P Z$, and leg muscle, liver, pectoralis, heart and brain had low expression levels of $C / E B P Z(P<0.01$, Supplementary Fig. 3$)$.

\section{Expression pattern of $C / E B P Z$ in visceral adipose tissue during chicken development}

The expression pattern of $C / E B P Z$ in the abdominal fat tissues of 1to 12 -week-old broilers from NEAUHLF was studied by real-time $P C R$. The results showed that $C / E B P Z$ was expressed in all the abdominal fat tissues tested. The relative $C / E B P Z$ expression data (C/EBPZ /ACTB) did not conform to a normal distribution (Shapiro-Wilk test; $W=0.92498, P<0.0001$ ). Scherier-Ray-Hare test showed that $C / E B P Z$ transcripts significantly associated with age $(H=33.560, P=0.00043)$ and broiler line (selected by high and low abdominal fat content, respectively; $H=8.949, P=$ 0.00278 ), and there was no significant association of $C / E B P Z$ transcripts with the interaction of age and line $(H=10.822, P=$ 0.45826).

Wilcoxon rank sum test showed that the $C / E B P Z$ expression of adipose tissue was significantly greater in lean broilers than in fat broilers ( $W=927, P=0.002578$; Fig. $2 A)$. Duncan's multiple tests showed that the C/EBPZ expression decreased during development, and it peaked at 1 week of age and reached its nadir at 12 weeks of age $(P<0.01$; Fig. $2 \mathrm{~B})$.

Additionally, at the ages of 7 and 9 weeks, the C/EBPZ expression was significantly greater in lean males than in fats $(P<0.05$; Fig. 2C). No significant difference was detected at the other ages $(P>0.05$, Fig. $2 C)$.

\section{RNA-seq analysis of chicken preadipocytes with C/EBPZ overexpression}

To investigate the role of $C / E B P Z$ in chicken adipose tissue, $C / E B P Z$ was overexpressed in chicken preadipocytes by transfection with the plasmid of pCMV-HA-C/EBPZ for $48 \mathrm{~h}$. Western blot analysis showed that chicken preadipocytes transfected with pCMV-HA-C/ $E B P Z$ for $48 \mathrm{~h}$ could express a HA-tag protein around $130 \mathrm{kD}$, and no similar protein was detected at this level in the cells transfected with plasmid of pCMV-HA (empty vector, EV; Fig. 3A).

RNA-seq was employed to study the effect of C/EBPZ overexpression on mRNA transcriptome of chicken preadipocytes (Fig. $3 \mathrm{~B})$, and the reads per kilobase of exon model per million mapped reads (FPKM) were got for expression analysis (Fig. 3C).

Totally, the valid data of 17927 genes were obtained, and negative binomial distribution test showed that there were 448 genes upregulated and 354 genes downregulated in the $C / E B P Z$ overexpressed preadipocytes compared with EV group $(P<0.05$; Fig. 3D and Supplementary Table 3), and the FPKM of C/EBPZ was significantly greater in C/EBPZ-overexpressed cells than in $\mathrm{EV}$ group $(P<0.05$; Fig. 3E).

Real-time PCR was employed to verify the reliability of the results of RNA-seq. The results showed that all the four genes tested were differentially expressed between EV and C/EBPZ group (Fig. 3F), consistent with the result of RNA-seq.

Gene Ontology (GO) enrichment analysis showed that the differential expression genes were enriched in 8 of GO terms at level of adjusted $P$ value $<0.05$ (Supplementary Table 4), namely, regulation of cellular ketone metabolic process (GO:0010565), negative regulation of protein serine/threonine kinase activity (GO:0071901), negative regulation of protein modification process (GO:0031400), negative regulation of MAPK cascade (GO:0043409), 
(A)

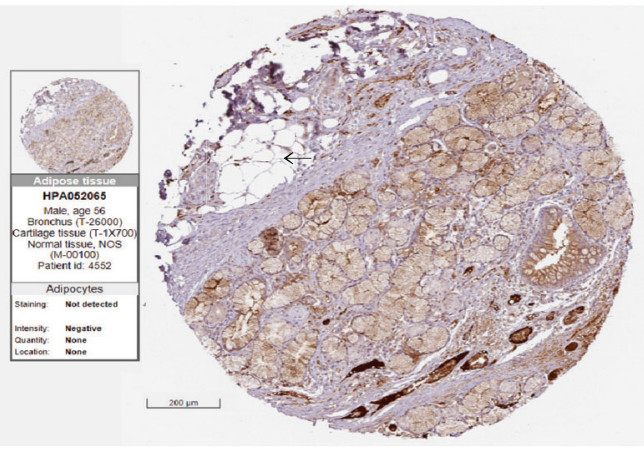

(C)

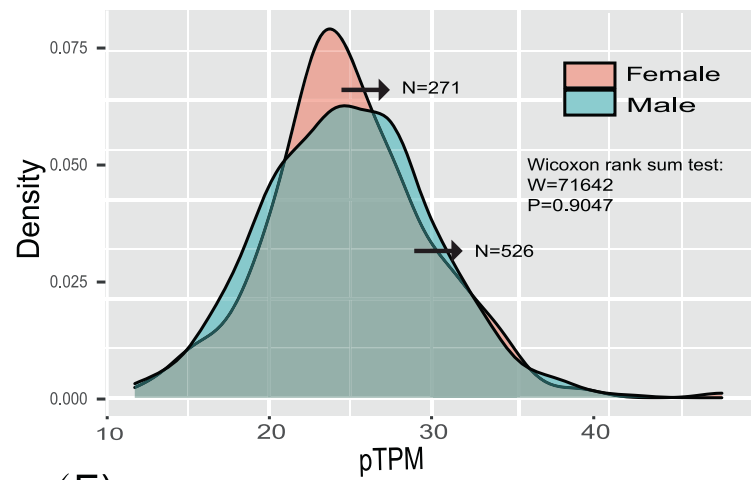

(E)

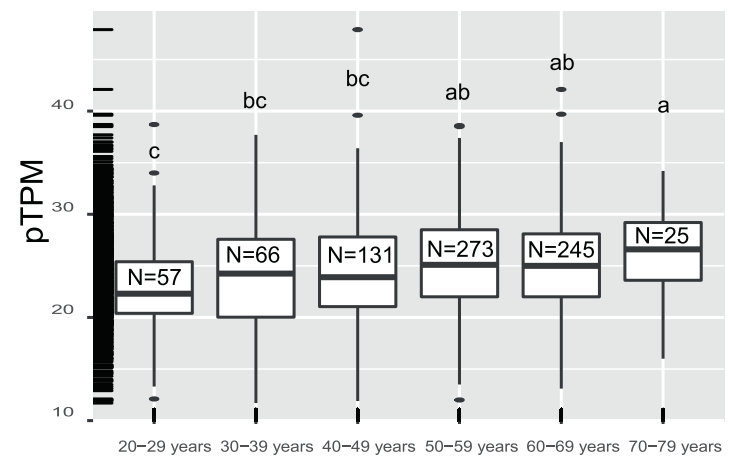

Age
(B)

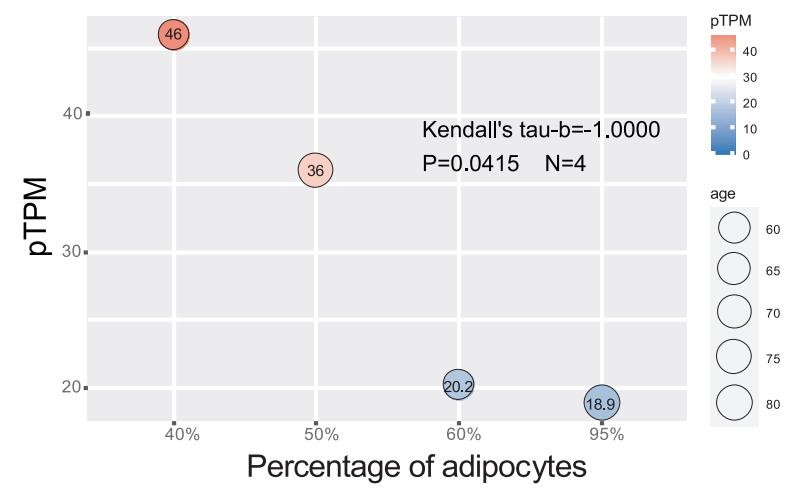

(D)

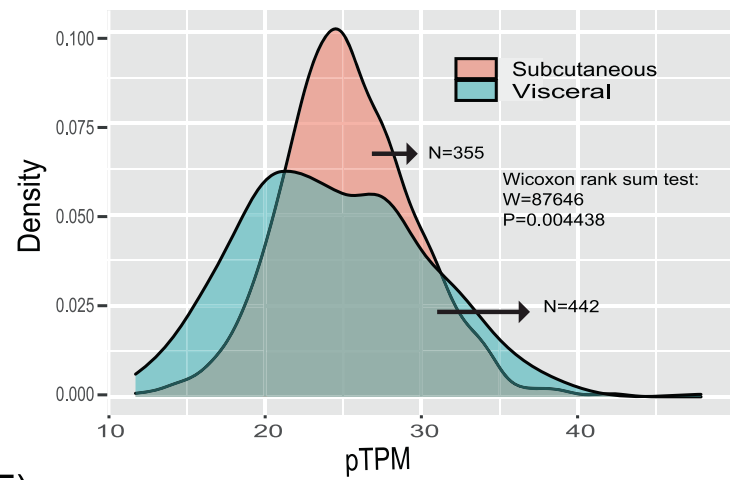

(F)

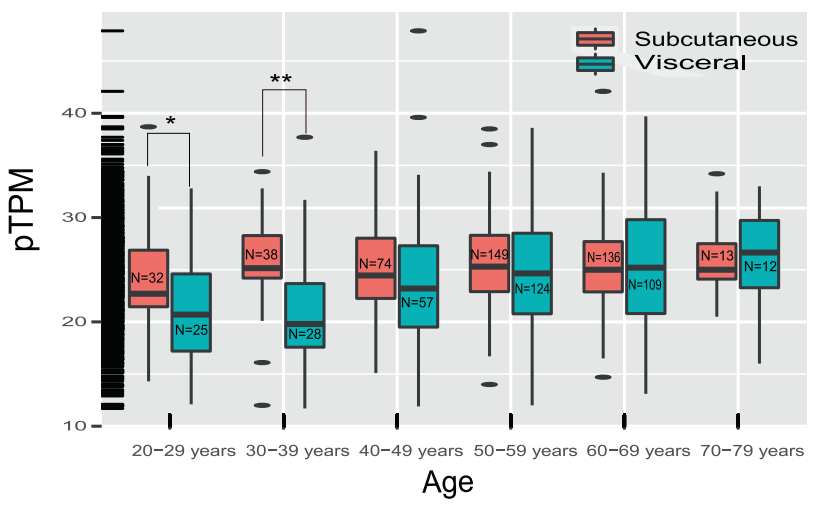

Fig. 1 Expression characterization of C/EBPZ in human abdominal fat tissue. The C/EBPZ expression data of human adipose tissues were achieved from the human protein atlas (https://www.proteinatlas.org/). A Immunohistochemical analysis of $C / E B P Z$ expression in adipose tissue $(\leftarrow)$. B Relationship analysis of $C / E B P Z$ expression and the percentage of adipocytes in adipose tissue. C The expression pattern of $C / E B P Z$ in the adipose tissues of males and females. D The expression pattern of C/EBPZ in subcutaneous and visceral adipose tissues. E The expression pattern of $C / E B P Z$ in adipose tissues at various ages. The different lowercase letters indicate statistically differences among ages (Duncan's multiple test, $P<0.05$ ). $\mathbf{F}$ The expression of $C / E B P Z$ in adipose tissues of the given location and age. Asterisks indicate significant differences between location at given age (Wilcoxon rank sum test), $P<0.05\left(^{*}\right), P<0.01\left(^{* *}\right)$.

Table 4. Percent identity matrix - created by Clustal2.1.

\begin{tabular}{|c|c|c|c|c|c|c|}
\hline & $\begin{array}{l}\text { Zebrafish } \\
\text { (Danio rerio) }\end{array}$ & $\begin{array}{l}\text { Tropical clawed frog } \\
\text { (Xenopus tropicalis) }\end{array}$ & $\begin{array}{l}\text { Chicken (Gallus } \\
\text { gallus) }\end{array}$ & $\begin{array}{l}\text { Human (Homo } \\
\text { sapiens) }\end{array}$ & $\begin{array}{l}\text { Rat (Rattus } \\
\text { norvegicus) }\end{array}$ & $\begin{array}{l}\text { Mouse (Mus } \\
\text { musculus) }\end{array}$ \\
\hline NP_001120886 & 57.49 & 100.00 & 59.92 & 61.17 & 60.19 & 60.58 \\
\hline NP_005751 & 57.01 & 61.17 & 64.60 & 100.00 & 82.47 & 82.20 \\
\hline NP_001102171 & 57.54 & 60.19 & 64.68 & 82.47 & 100.00 & 93.88 \\
\hline
\end{tabular}



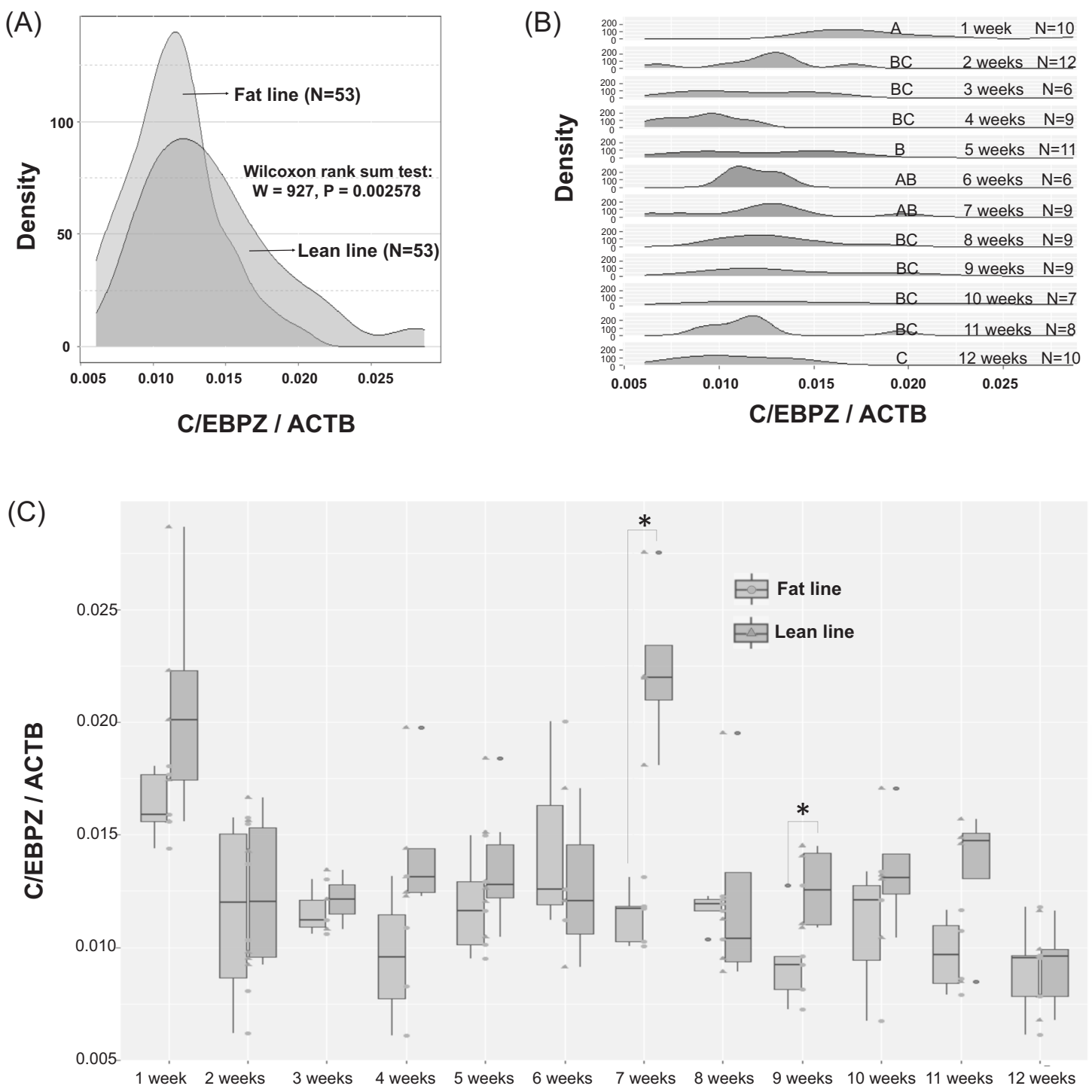

Age

Fig. 2 Expression characterization of C/EBPZ in abdominal fat tissue of NEAUHLF broiler lines. C/EBPZ mRNA expression in abdominal fat tissue of male broilers at various ages (each age, each line, $n=3-6)$ was analyzed by real-time RT-PCR. Chicken $\beta$-actin (ACTB) was used as internal control. A The kernel density of $C / E B P Z$ expression in abdominal adipose tissue of fat and lean birds. B The kernel density of $C / E B P Z$ expression in abdominal adipose tissue at various ages. The different uppercase letters indicate statistically differences among ages (Duncan's multiple test, $P<0.01$ ). C The expression of $C / E B P Z$ in abdominal adipose tissue of the given age and line. Asterisks indicate significant differences between fat and lean broilers at given age (Wilcoxon rank sum test or Student's $t$ test), $P<0.05\left(^{*}\right)$.

modulation by virus of host morphology or physiology (GO:0019048), modification by symbiont of host morphology or physiology(GO:0044003), negative regulation of MAP kinase activity (GO:0043407), and inactivation of MAPK activity (GO:0000188, Fig. 3G).

Additionally, Gene Set Enrichment Analysis (GSEA) showed that 729 and 99 gene sets of GO terms were significantly enriched in C/ EBPZ-overexpressed cells and EV group, respectively $(P<0.01$, Supplementary Tables 5-6). Among them, the gene set related to the adipose tissue development (GO:0060612) was significantly upregulated in C/EBPZ-overexpressed cells, and the gene set related to RNA polymerase II basal transcription factor binding (GO:0001091) was significantly upregulated in EV group (Fig. 3I). However, none of them was significantly enriched at FDR $<25 \%$.

The Kyoto Encyclopedia of Genes and Genomes (KEGG) analysis showed that C/EBPZ was involved in the DNA replication (GGA03030), Glycosaminoglycan biosynthesis (GGA00534/
GGA00532), Autophagy (GGA04136), and Arginine and proline metabolism (GGA00330) $(P<0.05$, Fig. $3 \mathrm{H}$ and Supplementary Table 7). However, none of them reach the level of adjusted $P$ value $<0.05$.

Additionally, GSEA analysis showed that 16 and 13 gene sets of KEGG pathways were significantly enriched in the C/EBPZoverexpressed cells and EV group, respectively $(P<0.01$, Supplementary Tables 8-9), and two gene sets were significant enriched at $F D R<25 \%$. They were ERBB signaling pathway (GGA04012) which upregulated in C/EBPZ-overexpressed cells and fatty acid degradation (GGA00071) which upregulated in EV group, respectively (Fig. 3J).

\section{The expression of $C / E B P Z$ in chicken preadipocytes during differentiation}

The expression pattern of $C / E B P Z$ during the differentiation of chicken preadipocytes induced by oleate was studied using 
(A)

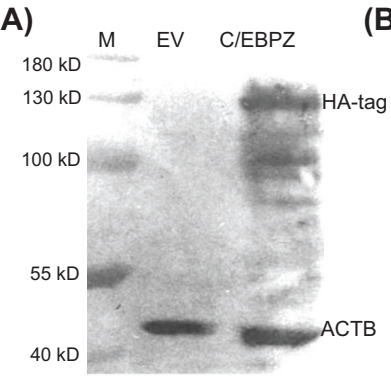

(D)

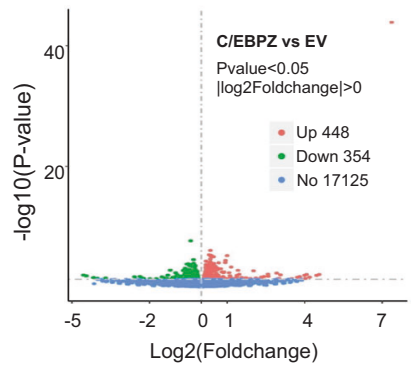

(F)
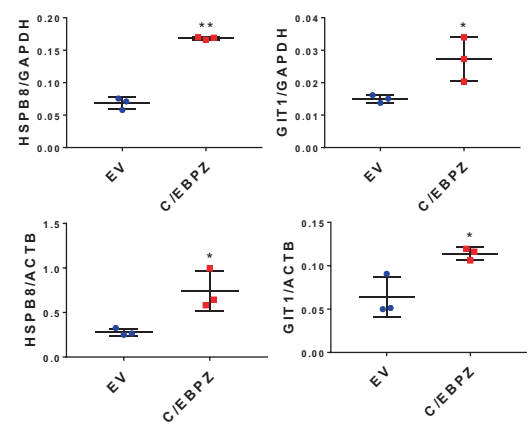

(G)

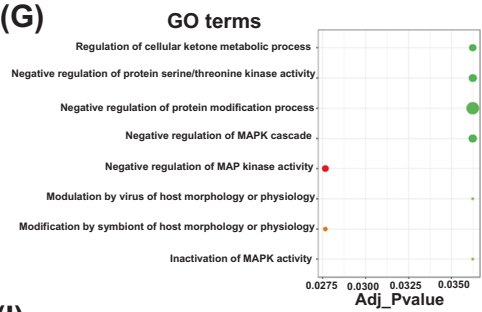

(l)

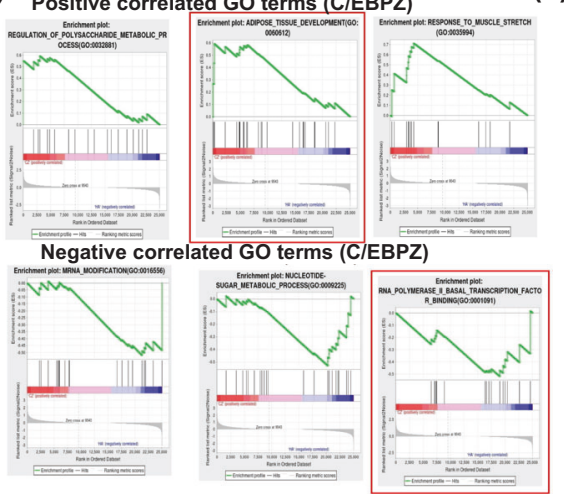

real-time $P C R$. The result showed that $C / E B P Z$ was expressed in chicken preadipocytes at every time point detected. Shapiro-Wilk tests showed that the data conform to a normal distribution $(P>0.05)$. Student's $t$ tests showed that there was no significant difference of $C / E B P Z$ expression at any time point

(E)

(J)
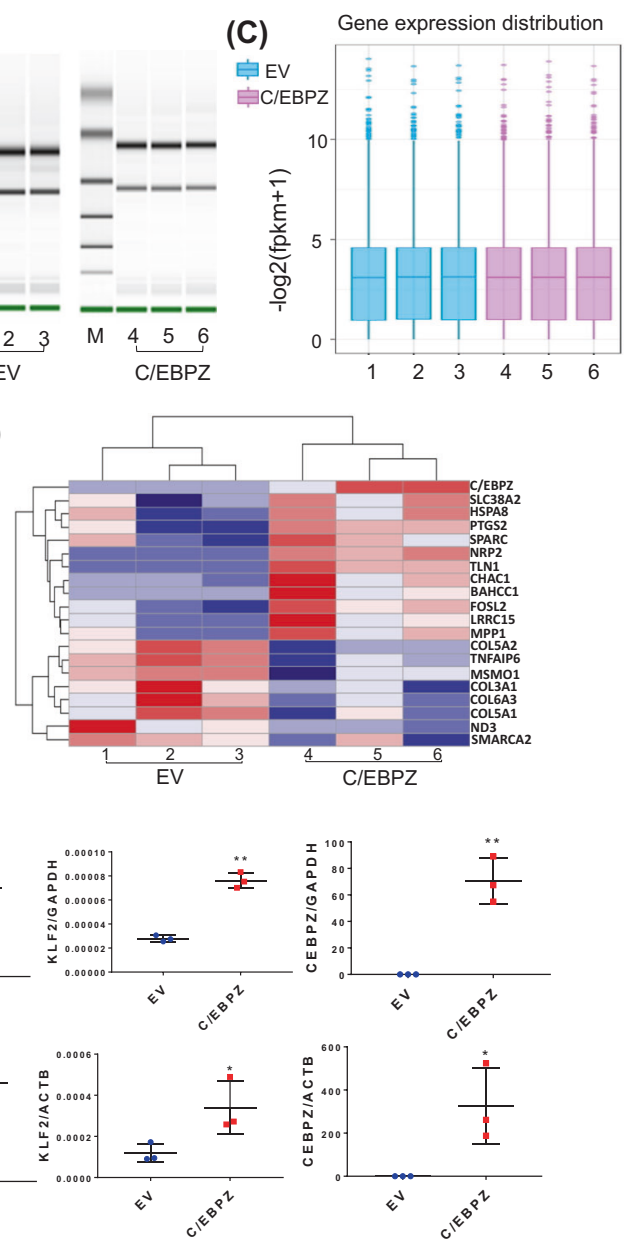

(H)
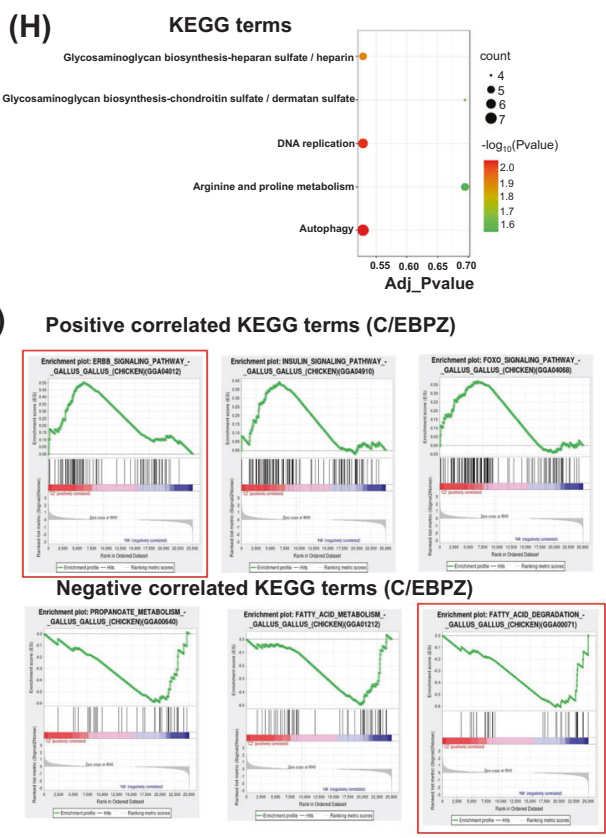

between negative control (NC) and preadipocytes induced by oleate $(P>0.05$; Fig. $4 A)$. Using the average expression level of $C / E B P Z$ of $N C$ at each time point to normalize the expression of $C / E B P Z$ in preadipocytes induced by oleate, and Duncan's multiple tests showed that the normalized expression of $C / E B P Z$ 
Fig. 3 RNA-seq analysis of chicken preadipocytes with C/EBPZ overexpression. A Western blot analysis of chicken preadipocytes transfected with pCMV-HA-C/EBPZ for $48 \mathrm{~h}$. B The quality control of total RNA extraction used for RNA-seq. C The summary of reads per kilo base of exon model per million mapped reads (FPKM) for genes got for expression analysis. D Differentially expressed genes between the C/ EBPZ-overexpressed (C/EBPZ) and empty vector-transfected (EV) cells. Negative binomial distribution tests showed that there were 448 genes upregulated and 354 genes downregulated in the C/EBPZ overexpressed preadipocytes. E Heatmap demonstrating the FPKM of 20 genes in six samples studied. $\mathbf{F}$ Expression analysis of differential expressed genes of RNA-seq using real-time reverse transcription-PCR. GAPDH and $A C T B$ were used as the internal controls. The scatter plot shows the quantification of given gene expression of 3 biologic replicates. Asterisks indicate significant differences (Student's $t$ test): ${ }^{*} P<0.05$ and ${ }^{*} P<0.01$. G Gene Ontology (GO) terms enrichment analysis of differential expressed genes. The enriched GO terms and adjusted $P$ levels were showed, and the size of bubble dot denoted the amount of genes in a certain term. H Kyoto Encyclopedia of Genes and Genomes (KEGG) pathway enrichment analysis of differential expressed genes. The enriched KEGG pathway terms and adjusted $P$ levels were showed, and the size of bubble dot denoted the amount of genes in a certain term. I-J Part of the results of Gene Set Enrichment Analysis (GSEA) of gene expression in the C/EBPZ-overexpressed cells and EV group.

(A)

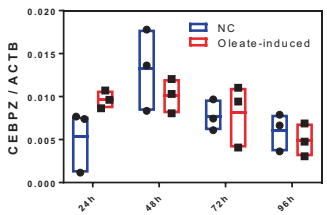

(C)

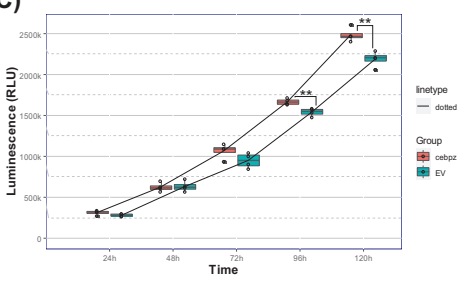

(E)

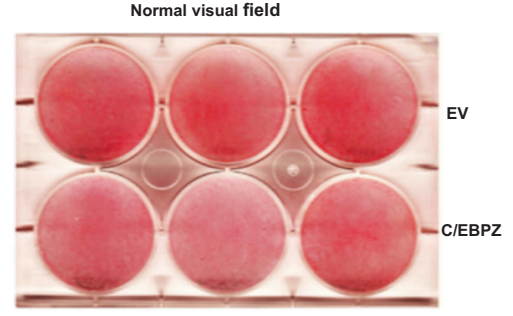

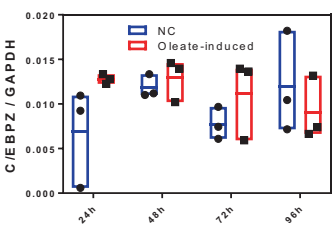

(D)

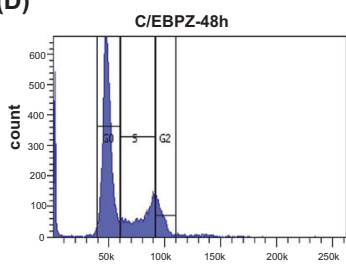

400xmagnification

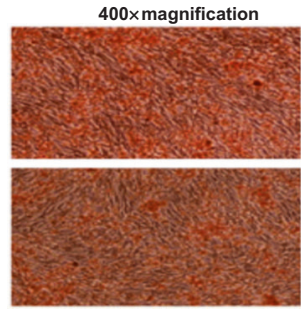

(B)
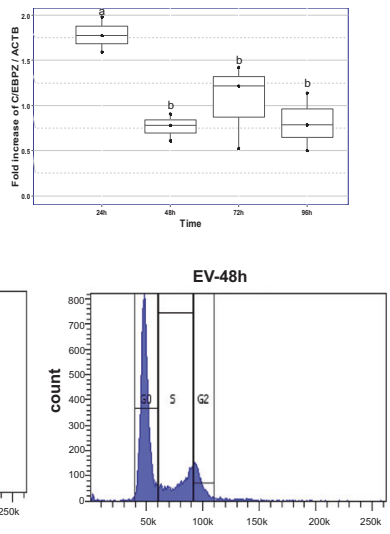

$(\mathrm{F})$

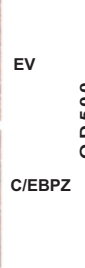

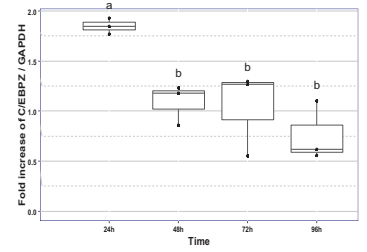

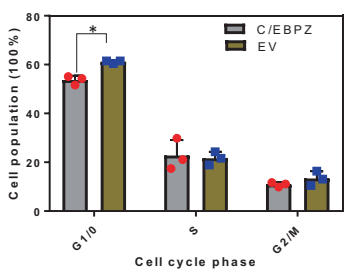

(G)

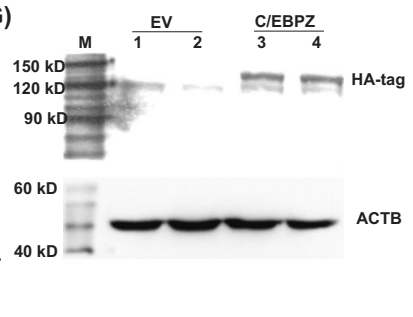

Fig. 4 Effect of C/EBPZ overexpression on the proliferation and differentiation of chicken preadipocytes in vitro. A The mRNA expression of $C / E B P Z$ during chicken preadipocyte differentiation. GAPDH and $A C T B$ were used as internal controls, and data are presented as boxplot of three replicates. B The relative expression of $C / E B P Z$ during chicken preadipocyte differentiation. The mRNA expression of $C / E B P Z$ at each time point was normalized to that of negative control (NC). The different lowercase letters above histograms indicate statistically significant differences among various time points (Duncan's multiple test, $P<0.05$ ). $C$ Effect of the overexpression of $C / E B P Z$ on chicken preadipocyte proliferation. The luminescent cell viability assay was performed in chicken preadipocytes transfected with $\mathrm{pCMV-HA-C/EBPZ}$ (C/EBPZ) or pCMV-HA (empty vector, EV), and data are presented as boxplot of four replicates. D Cell cycle analysis after overexpression of C/EBPZ in chicken preadipocytes for $48 \mathrm{~h}$. The diagram shows the percentage of cell population; and error bar represents the standard deviation of three replicates. E Oil red $\mathrm{O}$ staining of chicken preadipocytes induced to differentiate by oleate for $48 \mathrm{~h}$. $\mathbf{F}$ The lipid content of chicken preadipocytes measured by absorbance at $500 \mathrm{~nm}$. The diagram shows the absorbance at $500 \mathrm{~nm}$ of the extracted oil red O; and error bar represents the standard deviation of three replicates. G Western blot analysis demonstrated pCMV-HA-C/EBPZ could express C/EBPZ protein in chicken preadipocytes. C/EBPZ: cells transfected with pCMV-HA-C/EBPZ; EV: cells transfected with pCMV-HA; ${ }^{* P}<0.05$ (Student's $t$ test).

was significant decreased with the time of oleate induced, especially the $C / E B P Z$ expression at $24 \mathrm{~h}$ after oleate-induced was significantly greater than other time points $(P<0.05$; Fig. $4 B)$.

\section{Effect of C/EBPZ overexpression on chicken adipogenesis in vitro}

To further reveal the function of chicken C/EBPZ in adipogenesis, the effect of C/EBPZ overexpression on the proliferation and differentiation of chicken preadipocytes was studied in vitro. The result showed that C/EBPZ-overexpressing cells (Fig. 4G) exhibited an enhanced ability in cell proliferation at $96 \mathrm{~h}$ and $120 \mathrm{~h}$ after transfection compared to EV-transfected preadipocytes $(P<0.05$, Fig. $4 C)$. In addition, the flow cytometric analysis showed that after the transfection of $\mathrm{pCMV}-\mathrm{HA}-\mathrm{C} / E B P Z$ for $48 \mathrm{~h}$, the proportion of $\mathrm{G} 1$ phase cells in C/EBPZ-overexpressed preadipocytes was significantly lower than that of EV group $(P<0.05)$, while there was no significant difference in $S$ or G2 phase between them (Fig. 4D). Additionally, compared with EV-transfected chicken preadipocytes, C/EBPZ-overexpressing chicken preadipocytes exhibited a decrease in the intracellular lipid accumulation as evidenced by oil red $O$ staining (Fig. 4E) and a quantitative assessment $(P<0.05$, Fig. 4F).

Effect of C/EBPZ overexpression on the promoter activities of function genes of chicken adipose tissue

The effect of C/EBPZ overexpression on the promoter activities of several function genes of adipose tissue were studied using 
luciferase reporter assay. The results showed that the C/EBPZ overexpression significantly suppressed the promoter activities of $P P A R Y, C / E B P a, F A S N$, and $L P L$ in chicken preadipocytes $(P<0.05$, Fig. 5), and no significant effect on the promoter activity of GATA3 was found $(P>0.05$, Fig. 5). In addition, C/EBPZ overexpression significantly facilitated the promoter activities of chicken GATA2 and FABP4 in chicken preadipocytes $(P<0.05$, Fig. 5). Additionally, RT-PCR and western blot analysis showed that overexpression of C/EBPZ significantly inhibited PPARY expression in chicken preadipocytes $(P<0.05$, Fig. 6$)$.

\section{DISCUSSION}

\section{Expression of C/EBPZ in human adipose tissue}

The major functions of adipose tissue are to store and mobilize fat, and it plays important roles in the insulin resistance pathogenesis and development of T2DM [3]. C/EBPZ (Gene ID: 10153) is an interesting gene with a complex background of nomenclature [5], and its function in adipose tissue is not clear now.

Herein, to find out whether C/EBPZ plays a role in adipose tissue, the expression pattern of $C / E B P Z$ in human adipose tissue was studied using bioinformatics methods. The results showed that the C/EBPZ transcripts of human adipose tissue were negatively related to the percentage of adipocytes (Fig. 1), and they were negatively and positively associated with the transcripts of positive and negative regulators of adipogenesis, respectively (Supplementary Fig. 1), suggested that C/EBPZ might play a negative role in adipogenesis.

The ectopic fat hypothesis suggests that visceral adipose tissue confers more increased metabolic risk than subcutaneous adipose tissue [23]. The C/EBPZ transcripts were greater and more stable in subcutaneous adipose tissue than in visceral adipose tissue (Fig. 1), suggested that $C / E B P Z$ might be involved in different contribution of adipose tissue to metabolic risk. In addition, age changes in the metabolism of the adipose tissue and the part played by fatty tissue in decreasing the serum free fatty acid response [24]. It is possible that the increasing of $C / E B P Z$ expression of adipose tissue with age (Fig. 1), might be result from the metabolism change of adipose tissue, and C/EBPZ might be involved in the metabolic regulation of adipose tissue.

\section{Function of C/EBPZ in chicken adipose tissue}

$C / E B P Z$ was conserved in protein sequence and synteny pattern of chromosome between human and chicken, and both the C/EBPZ transcripts of human [20] and chicken (Supplementary Fig. 3) were ubiquitously expressed in various tissues, suggested that chicken and human C/EBPZs might have similar functions.

Chicken had been reported as an ideal animal model for obesity formation and metabolic disease [25]. In the current study, the results showed that $C / E B P Z$ transcripts of visceral adipose tissue were greater in lean broilers than in fat broilers and decreased during the growth of adipose tissue (Fig. 2), suggested that chicken C/EBPZ might be a negative regulator of visceral fat development, consistent with the finding in human.

The development of adipose tissue involves hyperplastic (proliferation) and hypertrophie growth (differentiation) of adipocytes, and histological analysis report showed that the difference in visceral adipose tissue between fat and lean birds at the age of 7 weeks was resulted from the different size and number of adipocytes [26]. The C/EBPZ expression of visceral adipose tissue was greater in lean males than in fat males at 7 weeks of ages (Fig. 2 ), it is possible that C/EBPZ regulated the differentiation or proliferation of adipocytes in vivo.

GSEA analysis of RNA-seq showed genes related to adipose tissue development (GO:0060612) were upregulated in C/EBPZoverexpressed chicken preadipocytes (Fig. 3), providing another evidence that C/EBPZ is a regulator of adipose tissue development. However, this was not consistent with the negative role of
C/EBPZ in adipogenesis. It is possible that multiple roles of C/EBPZ exited in adipose tissue. In addition, most adipocytes had been developed in chicken visceral adipose tissue at 7 weeks of age [26], C/EBPZ expression could be detected at all the tested ages from 1 to 12 weeks in chicken visceral adipose tissue and differentially expressed at 9 weeks of age between fat and lean birds, suggested that C/EBPZ might also play a role in developed adipose tissue.

Additionally, GSEA analysis of RNA-seq showed that genes associated with RNA polymerase II basal transcription factor binding (GO:0001091) were downregulated in C/EBPZ-overexpressed preadipocytes (Fig. 3), suggested that C/EBPZ could function as a transcription factor in chicken adipose tissue, consistent with the previous reports in human $[7,8]$.

The function of C/EBPZ in chicken adipogenesis was further studied in vitro, and the results showed that $C / E B P Z$ expression was decreased during differentiation of chicken preadipocytes (Fig. 4), which was similar as many other negative regulators of adipogenesis [3], for example, KLF2 [18] and KLF7 [19]. In addition, red $\mathrm{O}$ staining analysis showed that C/EBPZ overexpression inhibited the differentiation of chicken preadipocytes into adipocytes-like cells (Fig. 4). These results indicated that C/EBPZ was a negative regulator of chicken adipogenesis in vitro, too.

The adipogenesis is regulated by an elaborate network of transcription factors, and PPAR $\gamma$ oversee the entire terminal differentiation process at the center of this network [3]. In addition, C/EBPa could facilitated PPAR $y$ expression and adipogeneis [3], and GATA2 and FABP4 could attenuate adipogenesis through the suppression of PPARY expression [27, 28]. Luciferase reporter assays showed that C/EBPZ overexpression suppressed the promoter activities of chicken PPARY and C/EBPa, and promoted the promoter activities of chicken GATA2 and FABP4 (Fig. 5), further indicated that C/EBPZ was a negative regulator of adipogenesis. In addition, western blot analysis showed that C/ EBPZ overexpression inhibited PPARY expression (Fig. 6). Thus, mechanistically, C/EBPZ might inhibit chicken adipogenesis at least through the suppression of PPARY expression directly or indirectly (Fig. 6D).

LPL and FASN plays important roles in the transport of lipids [29] and de novo lipogenesis in adipose tissue [30], respectively. Overexpression of C/EBPZ inhibited the promoter activities of chicken FASN and LPL (Fig. 5), which was in line with the negative role of C/EBPZ in chicken adipose tissue development, suggested that C/EBPZ might also regulate fat accumulation of adipocytes (Fig. 6D). In addition, FABP4 acts as a fatty acids chaperone that regulates the basal and hormone-stimulated adipocyte fatty acid efflux [31]. Overexpression of C/EBPZ promotes the promoter activity of chicken FABP4 (Fig. 5), and the genes associated with fatty acid degradation (GGA00071) downregulated in C/EBPZoverexpressed cells (Fig. 3), shed a light on that C/EBPZ might play a role in the control of fatty acid efflux in adipocytes (Fig. 6D). Together, chicken C/EBPZ might also regulate the function of mature adipocytes in vitro.

The proliferation of preadipocytes is another important process of adipose tissue development [32]. KEGG analysis of RNA-seq showed that genes involved in DNA replication (GGA03030) were differentially expressed between C/EBPZ-overexpressed preadipocytes and EV group (Fig. 3), suggested that chicken C/EBPZ could regulate preadipocyte proliferation.

$\mathrm{C} / \mathrm{EBPa}$ is also a cell cycle regulator that promotes antiproliferation through inhibition of cyclin-dependent kinases 2, 4, and 6 and repression of S-phase gene transcription [33]. In the current study, C/EBPZ-overexpressed preadipocytes showed an increased proliferation ability (Fig. 4), and chicken C/EBPZ overexpression suppressed the promoter activities of $C / E B P a$ (Fig. 5), indicated that C/EBPZ could promote the proliferation of chicken preadipocytes (Fig. 6D). In addition, the flow cytometric analysis showed that the proportion of G1 phase 
(A)
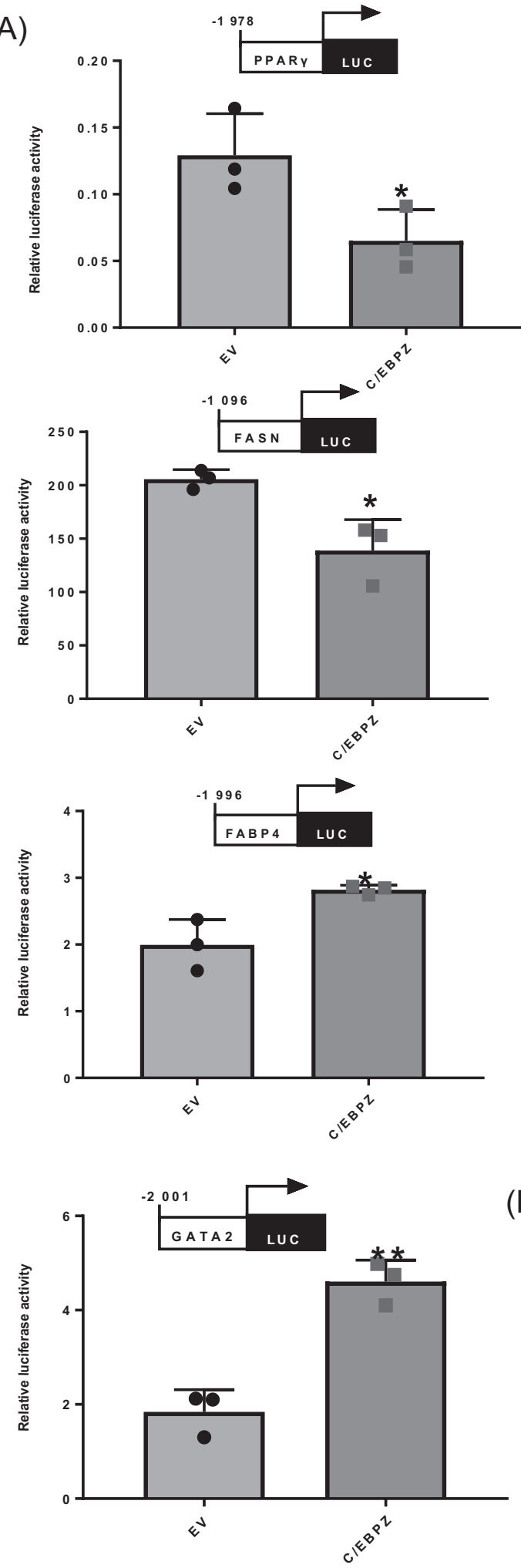
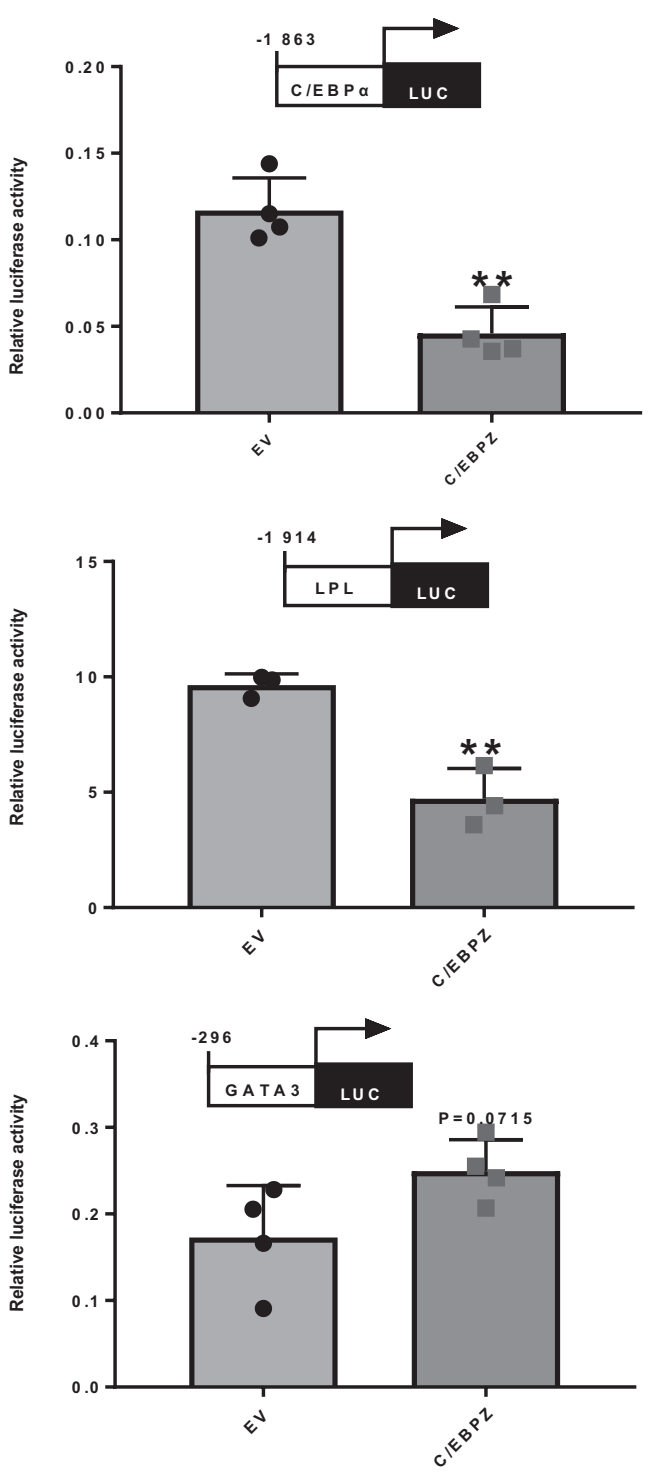

(B)

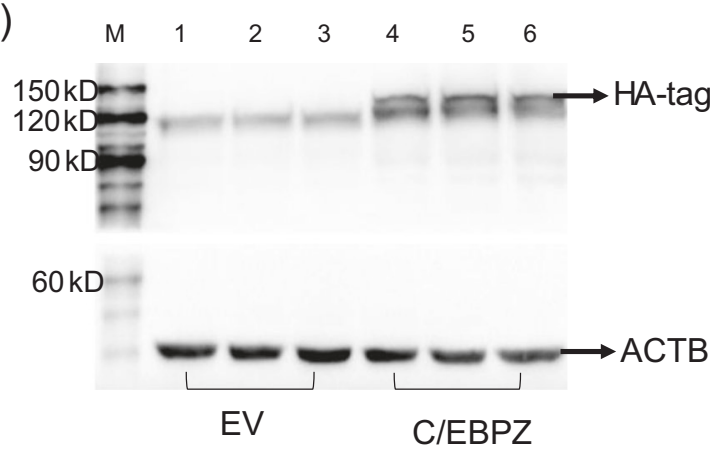

Fig. 5 Effect of C/EBPZ overexpression on the promoter activities of chicken PPARY, C/EBPa, FASN, LPL, FABP4, GATA2 and GATA3. A Luciferase reporter assays were conducted in chicken preadipocytes. The promoter activities were expressed as ratios of Firefly/Renilla luciferase activity, and data are presented as the mean \pm standard deviations of more than three replicates. B Western blot analysis of HA-tag protein in chicken preadipocytes. C/EBPZ cells transfected with $\mathrm{pCMV}-\mathrm{HA}-\mathrm{C} / \mathrm{EBPZ}$, EV cells transfected with $\mathrm{pCMV-HA}$; ${ }^{*} P<0.05 ;{ }^{* *} P<0.01$ (Student's $t$ test). 

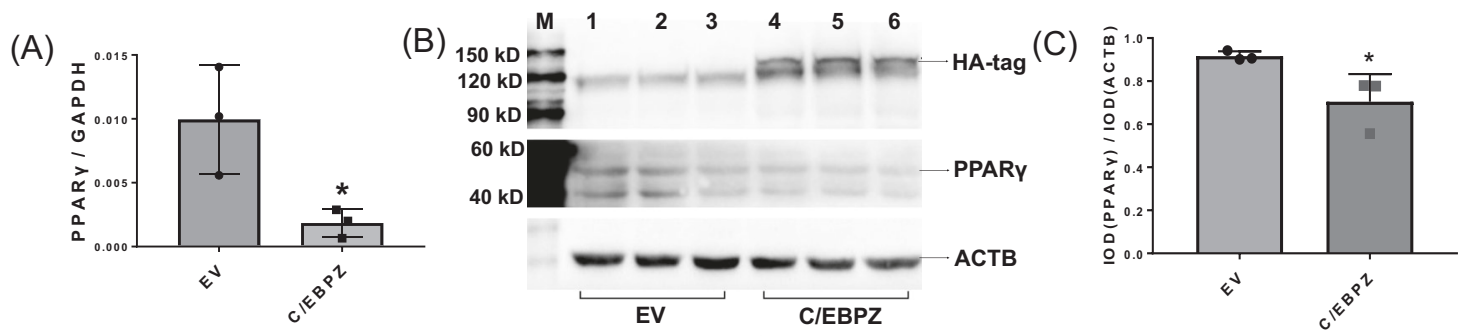

(D)

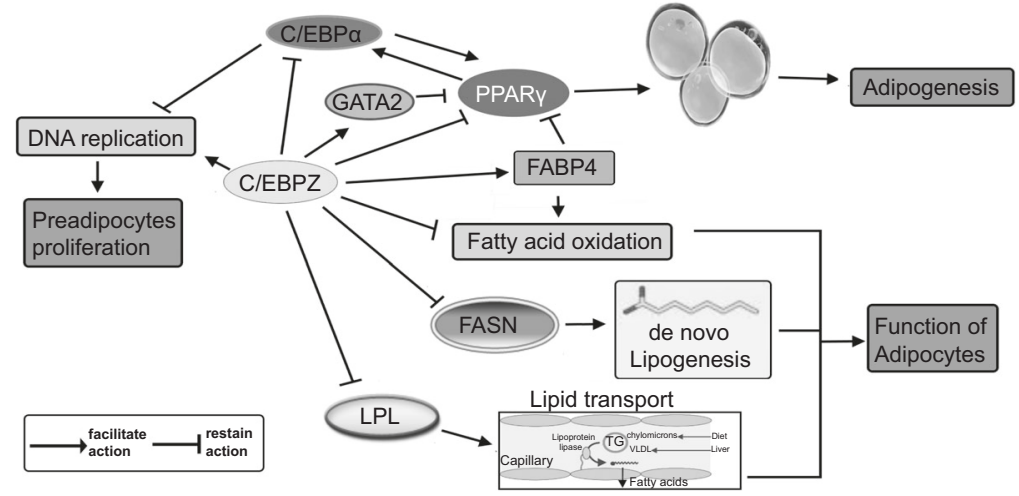

Fig. 6 Effect of C/EBPZ overexpression on the expression of PPAR $\boldsymbol{y}$ in chicken preadipocytes. A Real-time PCR analysis of $P P A R \gamma$ expression in chicken preadipocytes. Data are presented as the mean \pm standard deviations of three replicates. B Western blot analysis of PPAR $\gamma$ in chicken preadipocytes. C Histogram of expression of PPAR $\gamma$ normalized to the expression of ACTB in western blot analysis, and data are presented as the mean \pm standard deviations of three replicates. C/EBPZ: cells transfected with pCMV-HA-C/EBPZ; EV: cells transfected with pCMV-HA; ${ }^{*} P<0.05$ (Student's $t$ test). D Schematic summary about function and mechanism of chicken C/EBPZ in adipose tissue.

cells in C/EBPZ-overexpressed preadipocytes was significantly lower than that of EV group, demonstrated that C/EBPZ might improve preadipoyctes proliferation at least by inhibiting $C / E B P a$ expression and inducing the transition of cell cycle from G1 phase to S-phase.

GO analysis of RNA-seq showed that genes involved in the pathological process of infectious diseases (GO:0019048/ GO:0044003) were differentially expressed between C/EBPZ-overexpressed cells and EV group (Fig. 3G), which was consistent with the previous report that C/EBPZ took a part in the viral-human protein-protein interaction for SARS-CoV-1, MERS-CoV, and SARSCoV-2 [15]. In addition, the present study showed that C/EBPZ overexpression regulated adipogenesis, these shed a light on that further study on the biological function of C/EBPZ in adipose tissue might be of signification for revealing the molecular mechanism underlying the connection of metabolic diseases and infectious diseases, thereby to improve the prognosis of infectious diseases with metabolic diseases.

In conclusion, the current study provided novel insights into the mechanisms underlying development and function of adipose tissue and demonstrated that C/EBPZ might be a new regulator of the development and function of adipose tissue. The results of this study shed a light on revealing the molecular mechanism of adipogenesis and metabolic diseases.

\section{REFERENCES}

1. Kershaw EE, Flier JS. Adipose tissue as an endocrine organ. J Clin Endocrinol Metab. 2004;89:2548-56.

2. Chait A, Hartigh LD. Adipose tissue distribution, inflammation and its metabolic consequences, including diabetes and cardiovascular disease. Front Cardiovasc Med. 2020;7:22

3. Farmer SR. Transcriptional control of adipocyte formation. Cell Metab. 2006;4:263-73.

4. Mota DSP, Richard AJ, Hang H, Stephens JM. Transcriptional regulation of adipogenesis. Compr Physiol. 2017;7:635-74.

5. Pulido-Salgado $M$, Vidal-Taboada JM, Saura J. C/EBP $\beta$ and C/EBPS transcription factors: basic biology and roles in the CNS. Prog Neurobiol. 2015;132:1-33.
6. Tsukada J, Yoshida Y, Kominato Y, Auron PE. The CCAAT/enhancer (C/EBP) family of basic-leucine zipper (bZIP) transcription factors is a multifaceted highlyregulated system for gene regulation. Cytokine. 2011;54:6-19.

7. Lum LS, Sultzman LA, Kaufman RJ, Linzer DI, Wu BJ. A cloned human CCAAT-boxbinding factor stimulates transcription from the human hsp70 promoter. Mol Cell Biol. 1990;10:6709-17.

8. Imbriano C, Bolognese F, Gurtner A, Piaggio G, Mantovani R. HSP-CBF is an NF-Ydependent coactivator of the heat shock promoters CCAAT boxes. J Biol Chem. 2001;276:26332-9.

9. Castello A, Fischer B, Eichelbaum K, Horos R, Beckmann BM, Strein C, et al. Insights into RNA biology from an atlas of mammalian mRNA-binding proteins. Cell. 2012;149:1393-406.

10. Baltz AG, Munschauer M, Schwanhausser B, Vasile A, Murakawa Y, Schueler M, et al. The mRNA-bound proteome and its global occupancy profile on proteincoding transcripts. Mol Cell. 2012;46:674-90.

11. Andersen JS, Lyon $C E$, Fox $A H$, Leung $A K$, Lam $Y W$, Steen $H$, et al. Directed proteomic analysis of the human nucleolus. Curr Biol. 2002;12:1-11.

12. Barbieri I, Tzelepis K, Pandolfini L, Shi J, Millan-Zambrano G, Robson SC, et al. Promoter-bound METTL3 maintains myeloid leukaemia by $\mathrm{m}(6) \mathrm{A}$-dependent translation control. Nature. 2017;552:126-31.

13. Yao DM, Qian J, Lin J, Wang YL, Chen Q, Qian Z, et al. Aberrant methylation of CCAAT/enhancer binding protein zeta promoter in acute myeloid leukemia. Leukemia Res. 2011;35:957-60.

14. Ripke S, O'Dushlaine C, Chambert K, Moran JL, Sullivan PF. Genome-wide association analysis identifies 13 new risk loci for schizophrenia. Nat Genet. 2013;45:1150-9.

15. Gordon DE, Hiatt J, Bouhaddou M, Rezelj VV, Ulferts S, Braberg H, et al. Comparative host-coronavirus protein interaction networks reveal pan-viral disease mechanisms. Science. 2020;370:eabe9403.

16. Batchvarova N, Wang $X Z$, Ron $D$. Inhibition of adipogenesis by the stress-induced protein CHOP (Gadd153). Embo J. 1995;14:4654-61.

17. Zhang ZW, Sun YN, Rong EG, Li H, Wang N. Cloning, expression and function analysis of chicken FBXO38t1. Prog Biochem Biophys. 2013;40:845-58.

18. Zhang ZW, Rong EG, Shi MX, Wu CY, Sun B, Wang $Y X$, et al. Expression and functional analysis of Kruppel-like factor 2 in chicken adipose tissue. J Anim Sci. 2014;92:4797-805.

19. Zhang Z, Wang $H$, Sun $Y$, Li H, Wang N. Klf7 modulates the differentiation and proliferation of chicken preadipocyte. Acta Biochim Biophys Sin. 2013;45:280-8.

20. Uhlen M, Fagerberg L, Hallstrom BM, Lindskog C, Oksvold P, Mardinoglu A, et al. Proteomics. Tissue-based map of the human proteome. Science. 2015;347:1260419. 
21. Caldwell RB, Kierzek AM, Arakawa H, Bezzubov Y. Full-length cDNAs from chicken bursal lymphocytes to facilitate gene function analysis. Genome Biol. 2004;6:R6.

22. Milkereit $P$, Strauss D, Bassler J, Gadal O, Kühn H, Schütz S, et al. A Noc complex specifically involved in the formation and nuclear export of ribosomal $40 \mathrm{~S}$ subunits. J Biol Chem. 2003;278:4072-81.

23. Porter SA, Massaro JM, Hoffmann U, Vasan RS, O'Donnel CJ, Fox CS. Abdominal subcutaneous adipose tissue: a protective fat depot? Diabetes Care. 2009;32:1068-75.

24. Jelinkova M, Stuchlikova E. Age changes in the metabolism of the adipose tissue. Exp Gerontol. 1968;3:193-5.

25. Mellouk N, Rame C, Barbe A, Grandhaye J, Froment P, Dupont J. Chicken is a useful model to investigate the role of adipokines in metabolic and reproductive diseases. Int J Endocrinol. 2018;2018:4579734.

26. Guo L, Sun B, Shang Z, Leng L, Wang Y, Wang N, et al. Comparison of adipose tissue cellularity in chicken lines divergently selected for fatness. Poult Sci. 2011;90:2024-34.

27. Qiang T, Dalgin $G, X u H$, Ting CN, Leiden JM, Hotamisligil GS. Function of GATA transcription factors in preadipocyte-adipocyte transition. Science. 2000;290:134-8.

28. Garin-Shkolnik T, Rudich A, Hotamisligil GS, Rubinstein M. FABP4 attenuates PPARY and adipogenesis and is inversely correlated with PPARY in adipose tissues. Diabetes. 2014;63:900-11.

29. Bensadoun A. Lipoprotein lipase. Annu Rev Nutr. 1991;11:217-37.

30. Song Z, Xiaoli AM, Yang F. Regulation and metabolic significance of de novo lipogenesis in adipose tissues. Nutrients. 2018;10:1383.

31. Smith AJ, San De Rs MA, Juhlmann BE, Hertzel AV, Bernlohr DA. Mapping of the hormone-sensitive lipase binding site on the adipocyte fatty acid-binding protein (AFABP). Identification of the charge quartet on the AFABP/aP2 helix-turn-helix domain. J Biol Chem. 2008;283:33536-43.

32. Kulenkampff $E$, Wolfrum C. Proliferation of nutrition sensing preadipocytes upon short term HFD feeding. Adipocyte. 2019;8:16-25.

33. Johnson PF. Molecular stop signs: regulation of cell-cycle arrest by C/EBP transcription factors. J Cell Sci. 2005;118:2545-55.

\section{ACKNOWLEDGEMENTS}

This work was supported by the grant from the National Natural Science Foundation of China (Nos. 31960647 and 31501947), the Youth Innovative Talents Project of Shihezi University (No. CXBJ201905) and the Program for High-level personnel start at Shihezi University (No. RCZX201552). We thank Professor H. Li for the generous offer of abdominal adipose tissues of the 14th generation population of NEAUHLF.

\section{AUTHOR CONTRIBUTIONS}

$\mathrm{ZZ}$ conceived the project and designed experiments. ZZ, YC and XP performed bioinformatics analysis and data analysis. YC, LG, TL, QG and JC performed the molecule or cell biology experiments. $\mathrm{YZ}, \mathrm{XW}$ and $\mathrm{ZL}$ constructed and prepared plasmids. $Z Z$ and $Y C$ wrote the manuscript.

\section{COMPETING INTERESTS}

The authors declare no competing interests.

\section{ADDITIONAL INFORMATION}

Supplementary information The online version contains supplementary material available at https://doi.org/10.1038/s41366-021-01020-z.

Correspondence and requests for materials should be addressed to Zhiwei Zhang.

Reprints and permission information is available at http://www.nature.com/ reprints

Publisher's note Springer Nature remains neutral with regard to jurisdictional claims in published maps and institutional affiliations. 